\title{
ANALYSIS OF ACCESSIBILITY OF URBAN ROADS BASED ON SPACE SYNTAX AND DISTANCE MEASUREMENT
}

\author{
Zhuguang Pan ${ }^{1,2}$, Guiwen Lan ${ }^{1,2, *}$, Donglin Fan ${ }^{1,2}$, Yongliang Du ${ }^{1,2}$, Yi Zeng ${ }^{1,2}$ \\ ${ }^{1}$ Guangxi Key Laboratory of Spatial Information and Geomatics, Guilin University of Technology, Jiangan Road, Guilin, China - \\ (2277647553, 23955461, 812060461, 291296038,375308707)@qq.com \\ ${ }^{2}$ College of Geomatics and Geoinformation, Guilin University of Technology, Jiangan Road, Guilin, China
}

KEY WORDS: Space Syntax; Accessibility Analysis; Axis Line; Distance Measurement Method

\begin{abstract}
:
Space syntax and distance measurement are significant theoretical methods for studying road accessibility. Space syntax deals with the topological relationship between road networks, but the length of the road is ignored. While distance measurement method only takes into account the factors such as distance, time, and economic costs when studying road accessibility. Both of them are defective in evaluating the accessibility of transportation networks, and there are a few of researches that consider the interaction between road axes lines. In this paper a method for traffic network accessibility evaluation is proposed by combining the space syntax theory and the distance measurement method, and considering the influence of the interaction between road axes lines at the same time. The method is successfully applied for evaluating the traffic network accessibility of Nanning in Guangxi. The research shows that: (1) the results of the analysis are compared horizontally with traffic conditions at different times in Nanning City, and the experimental results are nearly in line with the actual road conditions. (2) The areas with high road density are Liangfeng District, Qingxiu District and Xixiangtang District. (3) Nanning City's road network center is located at the intersection of Xixiangtang District, Xingning District, Qingxiu District and Jiangnan District; its traffic network distribution is the densest and the overall level of urban road accessibility is higher. This study provides a theoretical basis for the development of Nanning's transportation network and has the reference significance for further optimizing the transportation planning in Nanning.
\end{abstract}

\section{INTRODUCTION}

The theory of spatial syntactic was first put forward by Hanson and Hillier, who believed that the spatial layout of cities would have an impact on the way people travel (Liao, 2013). After plenty of practice experiment, Peponis et al. (1997) proposed a spatial segmentation method of replacing the axis with feature points. Professor Jiang (2015) applied the theory of space syntax to GIS for the first time, and proposed that space syntax could be applied to urban road structure analysis, pedestrian prediction and traffic flow prediction. The study of urban traffic accessibility can be traced back to the early 19th century, and its research progress is relatively slow due to the lack of systematic research. In the 1990s, with the wide introduction and rapid development of GIS, Turing machine and other technologies, the content of spatial syntactic research is becoming more and more abundant; research methods are constantly innovating, research models are gradually perfecting, and research conclusions are deepening (Liu et al., 2011).

However, there are shortcomings in the application of spatial syntactic theory and distance measurement method in the study of the accessibility of urban transportation networks. In studying spatial syntactic theory, the researchers less consider the effects of the interaction between the road axis, and ignore the high-grade road axis which can lead to the improvement of the accessibility of other types of road axis connected with it; Some researchers use distance measurement to take into account the influence factors in the study of accessibility, but ignore the width and speed of roads which can also directly have an important impact on road accessibility (Liang et al., 2016). This paper mainly solves the above problems through the analysis of the road traffic network in the research area with adding the influence factor of the interaction between the road axes to the spatial syntactic theory in order to achieve the purpose of adding distance measurement analysis. On this basis, some important analysis results are obtained by combining spatial syntactic theory with distance measurement and analyzing the topological connection characteristics of traffic networks (Zheng et al., 2008). This paper is of great significance to the planning and construction of the city, providing an optimal and cost-effective auxiliary design method with high predictability, which will play an important role in urban planning.

\section{URBAN ROAD ACCESSIBILITY RESEARCH METHOD}

\subsection{The Definition and Research Method of Accessibility}

Accessibility is defined as the ease with which one place can reach another (Li and Wang, 2017). Measurement accessibility of scholars at home and abroad mainly includes gravity measurement method, distance measurement method, topological measurement method, and cumulative opportunity method (Chen et al., 2017). Different methods correspond to their respective suitable subjects according to their own characteristics. The idea of gravity measurement method from the law of universal gravitation. The researchers believe that the spatial effect of cities decay with the increase of distance, and that it is a broader method to measure accessibility by combining the attributes of geographical entities with distance measurement is one of the most important methods for

\footnotetext{
Corresponding author
} 
measuring accessibility (Zhang et al., 2008), where distance can refer to time distance, space distance, cost distance (Yang and Zhou, 1999); Topological measurability is suitable for situations where the number of interchanges is more important than the actual distance and is commonly used in road, rail and aviation transport networks (Jin, 2001); The cumulative opportunity method evaluates the level of accessibility by assessing the convenience of people's travel (Chen et al, 2007). On the basis of the research object, this paper combines distance measurement method with topological measurement method, using the idea of graph theory, abstracts the traffic network into a topological network, and constructs an accessibility model from three aspects: spatial barrier, space function and topological connection to analyze the accessibility of road traffic network.

\subsection{Theory of Space Syntax}

Spatial syntax is a mathematical method that describes the structure and morphology of urban space, and belongs to the topological measurement method in the measurement of accessibility. It originated in architecture and later applied to GIS and computer science. Proceeding from the space itself, it explains the spatial form of the city system, the traffic network and the traffic flow (Sheng, 2012). Space syntax represents the interrelationship of space by connecting values, control values, local depth values, average depth values, total depth values, local integration values, global integration, and other parameter values.

1. The connection value represents the number of nodes connected to node in the axis diagram, and is generally represented by $\mathrm{Ci}$. It reflects the selectivity and connectivity of the axis in this space (Zhai, 2008). The higher the connection value, the better the spatial permeability of the axis.

2. The control value indicates the degree of control of the space with which the axis intersects, reflecting the importance of the axis to the entire space. Assuming that the weight of each node in the system is 1 , the weight assigned by a node a form the adjacent node $\mathrm{c}$ is $1 /$ (the connection value of $\mathrm{c}$ ), then the sum of the reciprocal of the connection value of the node directly connected to a is a The weight assigned to each adjacent node, which indicates the degree of mutual control between the nodes, so it is called the control value of the node. The higher the control value in the road axis diagram, the more important this axis is, that is, the calculation formula of the entire axis is

$$
\operatorname{ctrl}_{i}=\sum_{1}^{n} \frac{1}{C_{\mathrm{i}}}
$$

where $\quad c t r l=$ connection value

$$
\begin{aligned}
& C=\text { the connection value of } \mathrm{c} \\
& i=\text { number of nodes } \\
& n=\text { total number of nodes }
\end{aligned}
$$

3. The depth value represents the smallest connection tree from one-unit space to another in a space system. The depth value can be divided into a global depth value, a local depth value, and an average depth value, which are generally represented by $\mathrm{D}, \mathrm{LD}$, and $\mathrm{MD}$. The depth value is a non-scale distance variable, and it is not a fixed quantity. As the observer's viewpoint in the city is different, the distance between the near and far of the line of sight, and the step distance from small to large, the depth value will change. Let the shortest step distance from any point in space to any other point be $\mathrm{d}$ ( $\mathrm{d}$ is an integer), the minimum is 1 , and the maximum number of steps is $n$, where $1<\mathrm{d}<\mathrm{n}$, when $\mathrm{d}=1$, it indicates the specified node. The number of directly connected nodes, the depth value is a step depth value. When $d$ is slowly increased, the depth value of this phase is the local depth value. When $d$ is increased to the maximum value $n$, the depth value at this time is the global depth value. The depth value is not an independent morphological variable, but an intermediate variable for calculating the spatial form integration degree, which is expressed by the average depth in a specific application. Depth value is one of the most important concepts in space syntax. It expresses the accessibility of nodes in the topological sense, that is, the convenience of nodes in space systems, the formulas are as follows:

$$
\begin{aligned}
& D_{i}=\sum_{i=1, j \neq 1}^{n} d_{i j} \\
& L D_{i}=\sum_{i=1, j \neq i, d_{i j} \leq 3}^{n} d_{i j} \\
& M D_{i}=\frac{D_{i}}{n-1}
\end{aligned}
$$

where $\quad D=$ global depth value

$L D=$ local depth value

$M D=$ average depth value

$d=$ any point to any other point

$n=$ Total number of nodes

$i=$ number of nodes

$j=$ number of nodes

4. The integration value reflects the aggregation or dispersion degree of nodes and other nodes in the network, which can be divided into global integration degree (Ii) and local integration degree value (LIi). The overall integration value indicates the closeness of the node to all nodes in the whole system. The local integration value indicates how close the node is to the nodes in the nearby steps, usually calculating a three- or ten-step range. That is, the accessibility of the node in the topological sense, that is, the convenience of the node in the space system, seeing the following formulas:

$$
I_{i}=\frac{n-2}{2\left(M D_{i}-1\right)}
$$

$$
l I_{i}=\frac{n\left[\log _{2} \frac{n+2}{3}-1\right]+1}{(n-1)\left(M D_{i}-1\right)}
$$

where $\quad I=$ integration value

$l i=$ global integration degree

$M D=$ average depth value

$n=$ total number of nodes

$i=$ number of nodes 


\subsection{Distance Measurement}

Distance measurement is mainly measured by distance, time and economic cost. Although the distance measurement method is concise in practice, the single use distance, time, economic cost index measurement of universality is not comprehensive, and cannot accurately represent the region's universality. In view of the lack of practical application of distance measurement, many scholars have revised it in order to enhance the applicability and accuracy of distance measurement.

\subsection{Spatial Axis Segmentation Method}

Axis segmentation is the premise of applying space syntax analysis. The segmentation methods of space syntax are mainly view space, axis and convex polygons, and different segmentation methods correspond to different application scenarios (Shao, 2010). Space syntax method generally applies to axis method to realize the division of space. Space syntax provides that the entire spatial system is covered with the shortest axis, through each convex space, and then each axis is treated as a node, which is transformed into a diagram according to the transition between them, and various spatial variables are calculated and analysed, and the severity of the syntax variables are represented by different shades (Wang and
Wang, 2013), as shown in Figure 1 below, the detailed spatial segmentation process can be found here.
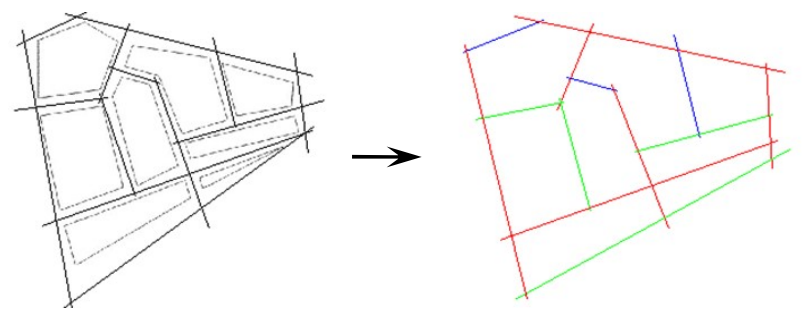

Figure 1. Spatial segmentation of axes

\section{ANALYSIS OF ROAD ACCESSIBILITY IN NANNING CITY}

\subsection{Data Sources}

The transportation network data set studies in this paper, and the advantages and disadvantages of Baidu Map, Gaode Map, national lying traffic map and Map World are compared. The traffic data of The Gaode Map can meet the needs of the Institute, and it is most convenient to digitize, so we chose the data provided by The Gode Map.

\begin{tabular}{|c|c|c|}
\hline Data sources & Advantage & Disadvantage \\
\hline National administrative district map & Relatively authoritative & $\begin{array}{c}\text { The extraction of linear data is difficult, } \\
\text { and the digitization process is relatively } \\
\text { complicated. }\end{array}$ \\
\hline Gaode Map & $\begin{array}{c}\text { It can be imported into ArcGIS directly } \\
\text { through ArcGIS plugin, which is } \\
\text { convenient for digitization. }\end{array}$ & $\begin{array}{c}\text { The extraction of linear data is difficult, } \\
\text { and the digitization process is relatively } \\
\text { complicated. }\end{array}$ \\
\hline Map World & $\begin{array}{c}\text { Relatively authoritative, can be directly } \\
\text { imported into ArcGIS through ArcGIS } \\
\text { plug-in, digital is relatively simple }\end{array}$ & $\begin{array}{c}\text { The data is relatively simple compared to } \\
\text { the high German map and Baidu map, } \\
\text { but the vector process is more likely to } \\
\text { cause the lack of data. }\end{array}$ \\
\hline Baidu Map & $\begin{array}{c}\text { Digital is more convenient, can be } \\
\text { combined with Baidu API interface to } \\
\text { facilitate the acquisition of POI data }\end{array}$ & $\begin{array}{c}\text { The authority is not as good as the } \\
\text { administrative map and the sky map } \\
\text { released by the country. }\end{array}$ \\
\hline
\end{tabular}

Table 1. Comparison of various types of map data

\subsection{Research Area Overview and Research Methods}

Nanning city is the political, economic and cultural center of Guangxi, it is the only way form place where the great arteries of the south-western China. Nanning city is a major gateway city for China's a leading city for ASEAN development and cooperation, the southwest sea transportation comprehensive transportation hub city, and the national "The Belt and Road" organic connection. Therefore, Nanning City is selected as the research area and it is representativeness ( $\mathrm{Ji}$ and $\mathrm{Su}, 2013$ ).

After selecting Nanning City as the research object, the traffic map is obtained as the basic data by loading the China Map software module in ArcGIS software, digitizing the raster map and getting the vector data map of Nanning City, and the spatial correction and projection transformation is processed, the road width and road speed are added to it. According to the width of the road to determine the level of the road, the resulting railway, national road, highway layer, weight value 4, 2, 4 is given; road speed in accordance with the relevant provisions of the traffic rules: the railway and highway is $80 \mathrm{~km} / \mathrm{h}$, the national highway is $60 \mathrm{~km} / \mathrm{h}$; The resulting axis map is a topological check to ensure that no isolated axis or arc segment is a prerequisite for calculating spatial syntactic model parameters, the Axwoman software module is used to calculate the spatial syntactic model indicators in the study area, finally, ArcGIS software is used to analyze the accessibility of road traffic networks, and visualize it. The main flowchart is shown in Figure 2. 


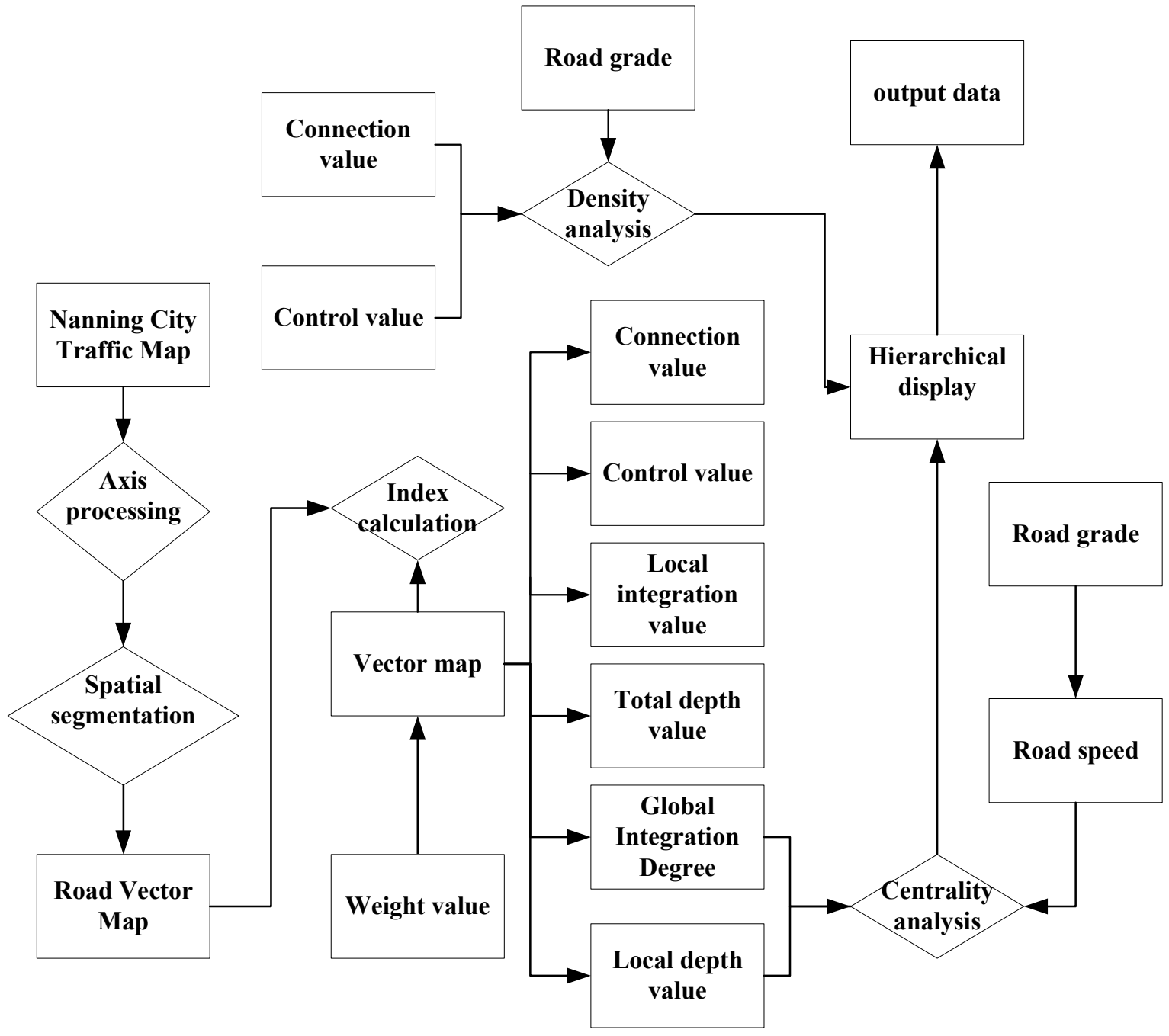

Figure 2. Data processing flowchart

\subsection{The Process and Results of Centrality Analysis}

The central analysis can be carried out to study the road network center and local center of Nanning City. The more central the area, the more convenient the area. The central analysis of the Nanning area in ArcGIS requires a data set with global integration values, total depth values, road levels, and road speed. The specific analysis process is as follows: loading the city vector map in ArcGIS software, according to the total depth value of the road network classification display, according to the characteristics of ArcGIS data, this paper converts linear data into point data, and generates the corresponding network diagram, to ensure the connectivity of the network diagram, According to the network analysis and cost matrix analysis module in ArcGIS, the actual distance is generated to obtain the actual road length, and then the central grid map is obtained by using nuclear density analysis, and the effect map of Nanning central analysis is output after adding the weight value in the attribute table. Figure 3 shows the central analysis of the railway and road in Nanning. Among them, the darker the color of the grid map indicates that the higher the center of the road network, the better access of these sections, the greater the traffic flow, which is more consistent with the comparative traffic flow status.

Figure 3 can be found that Nanning City's road network center is located in Xixiangtang district, Xingning District, Qingxiu District and Jiangnan District intersection. This intersection also condensed the main economic lifeblood of Nanning City. In Longan County, Mashan County, Shanglin County, Binyang County, Heng County did not realize the obvious center, the impact capacity is relatively weak, indicating that the construction of the road network in the region is relatively backward, these areas in the overall development process has been a certain degree of neglect. 


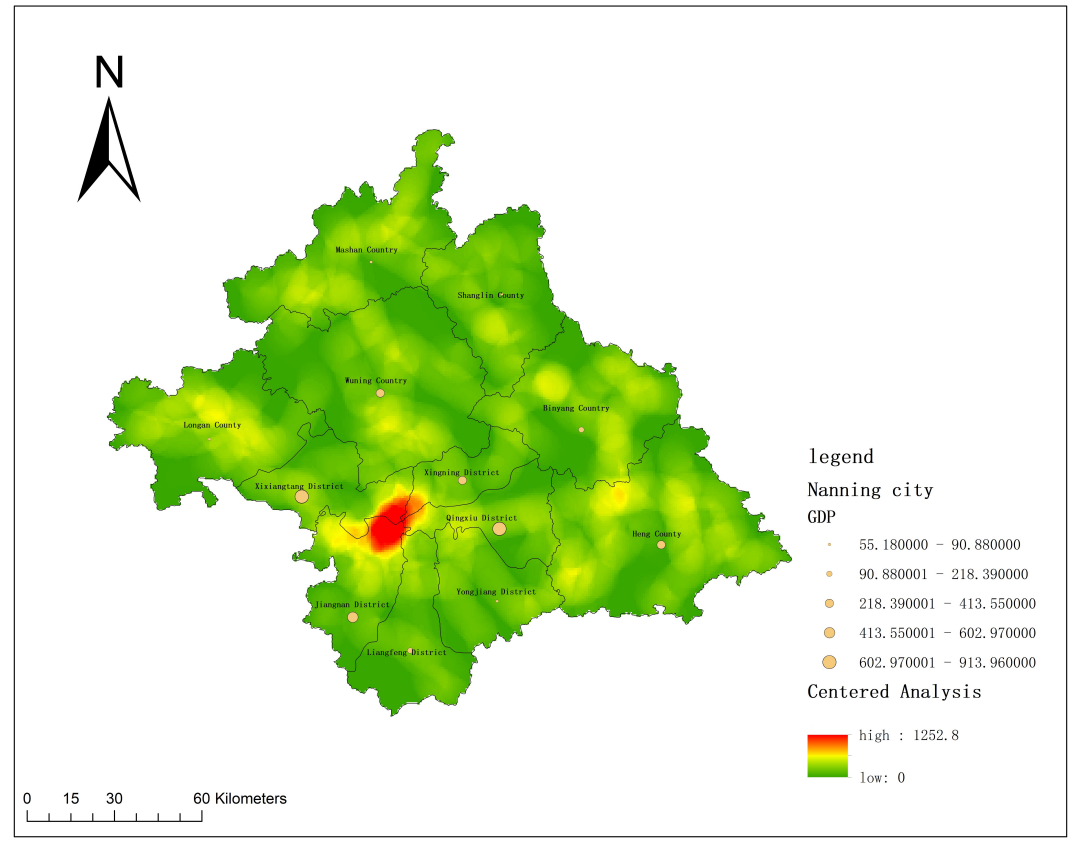

Figure 3. Analysis of road centrality in Nanning city

\subsection{Density Analysis Process and the Result Analysis}

Density analysis can study the density of the road network in Nanning City, and the higher density of the area indicates that more roads can be selected, the more convenient the road network traffic in this section of the road. The data needed for density analysis are the connection value, the control value and the weight value in the road grade, the corresponding grid map is generated after the line density analysis in ArcGIS, and the result chart of the analysis is output.

Figure 4 shows the density map of the main roads and railways in Nanning City, where the density of the road network is H- shaped, and these most concentrated traffic are called integrated nuclei, which are the link between the city center and the surrounding areas, and are the most active areas of human activity within the city, but also the areas prone to swarming. At present, the highest density of road network is located in Liangfeng District, Qingxiu District and Xixiangtang District, the current construction of the road network in the area has become saturated, but the construction of road network in Mashan County, Shanglin County, Wuming County, Xingning District and Yanning District of Nanning City needs to be further improved and developed.

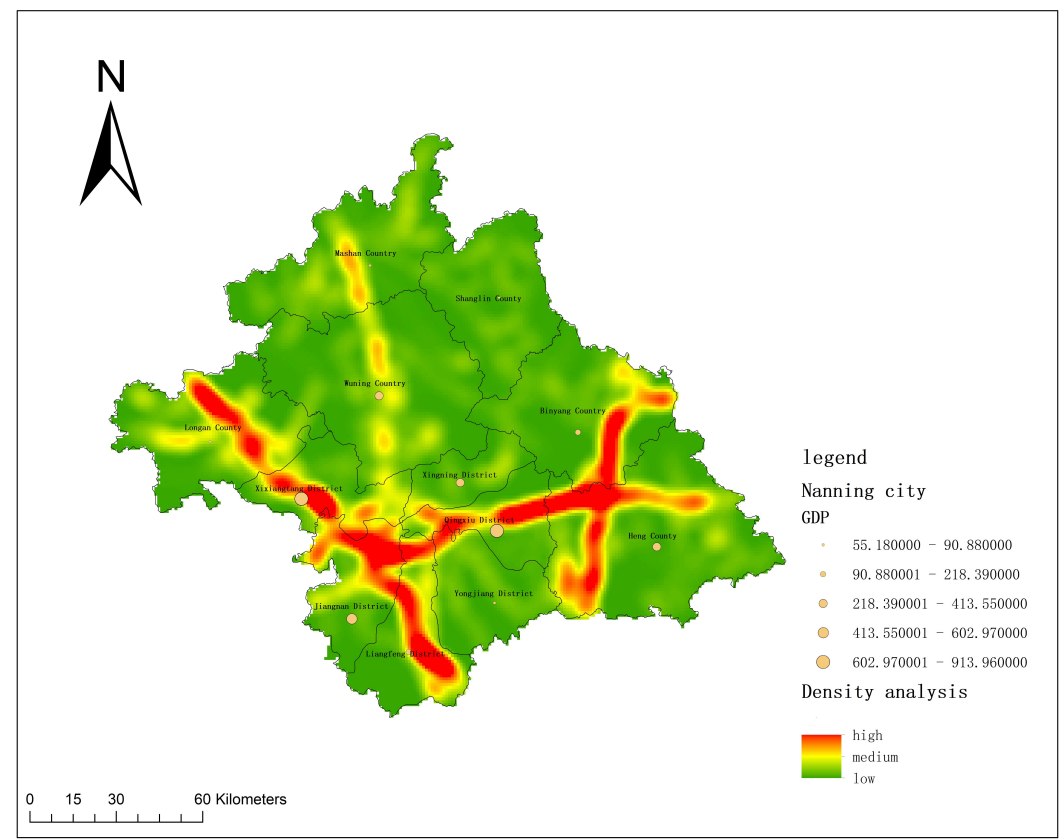

Figure 4. Analysis of road density in Nanning city 


\subsection{The Results of Accessibility Analysis}

Nanning City traffic accessibility level is generally high, the high and low level of accessibility with different colors of the axis, from blue to red indicates the level of accessibility from low to high. Figure 5 shows the positive correlation between the reach level of the road traffic network in Nanning City and the economic development. Roads with high accessibility include the Nankun Line, Xianggui Line, Guangkun Line, Southern Line, Guangkun Expressway, Nanning Huancheng Expressway, Lanhai Expressway, which drive the rapid development of the surrounding economy.

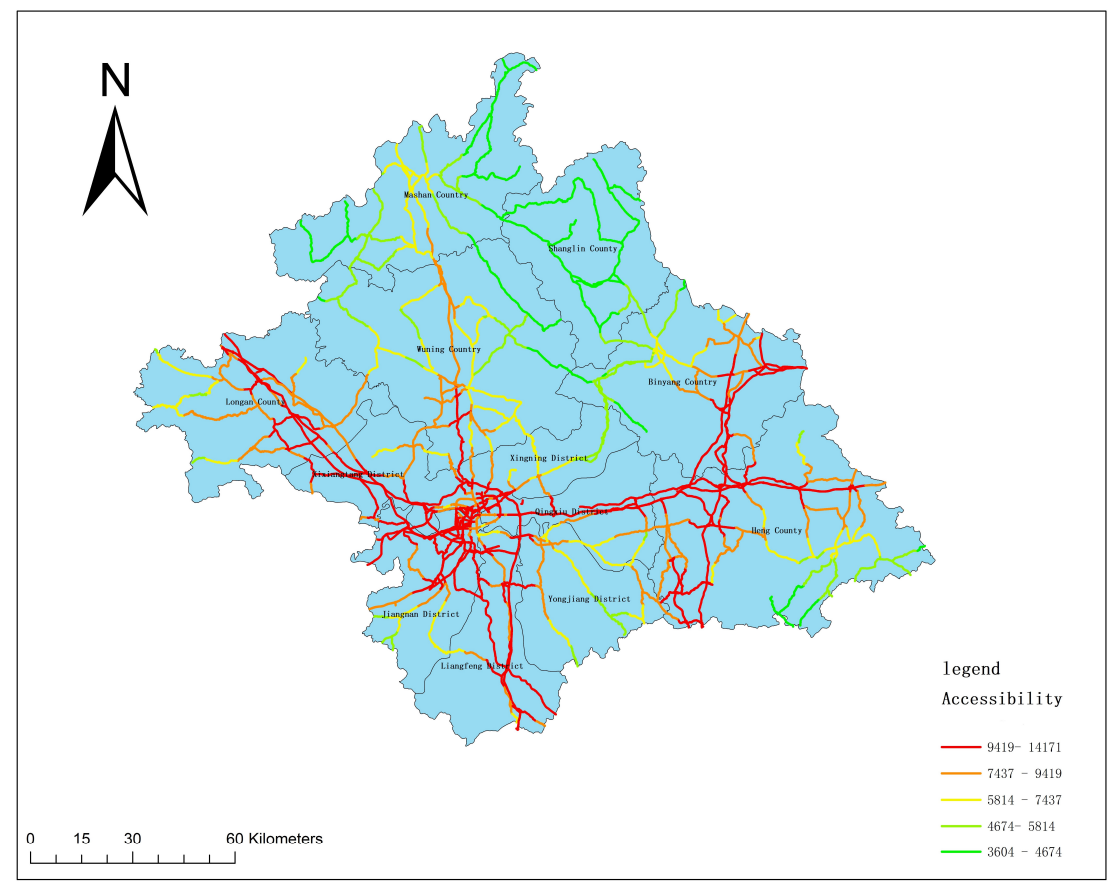

Figure 5. Road accessibility analysis of Nanning city

\section{CONCLUSION}

In this paper, the distance measurement method, space syntax and GIS are combined, and the road network in Nanning City is analyzed by density and centrality. With GIS as the platform, the space syntax provides a different spatial perspective for urban traffic analysis-based expression. The results show that space syntax has high predictability and provides an optimal and cost-effective design method for this paper. Furthermore, the results show that the road network center in Nanning city is located at the intersection of Xixiangtang District, Xingning District, Qingxiu District and Jiangnan District, with the trend of divergence in the area, and the other areas have not formed a clear centrality; in addition, the road network density is relatively high and the road network gradually matures, and the level of economic development is relatively high. Finally, the results of the analysis are compared horizontally with traffic conditions at different times in Nanning City, and the experimental results were more in line with the real road conditions, which further illustrate that the analytical method of this paper has a good effect on the study of the accessibility of urban road networks.

\section{ACKNOWLEDGEMENTS}

The research work is supported by National Natural Science Foundation of China under Grant No.41861050.

\section{REFERENCES}

Chen, J., Lu, F., Cheng, C. X., 2007. Review on accessibility measurement methods and application research progress. Progress in Geography, 26(5), 100-110.

Ji, Z., Su, Y. F., 2013. Nanning city: building Guangxi's "best district" and regional international cities. Money China, (3), 6869.

Jiang, B., 2015. Axwoman 6.3: an arcgis extension for urban morphological analysis. http://giscience.hig.se/binjiang/Axwoman/, University of Gävle, Sweden.

Jin, F. J., 2001. Development of China's air passenger flow network and its regional system research. Geographical Research, 20(1), 31-39.

Liao, M. Q., 2013. Research on the spatial distribution of Changsha city commercial center based on spatial syntax. Hunan University, Changsha, Hunan province, China.

Liu, C. L., Yu, R. L., Zeng, J. X., 2011. Spatial research progress of urban transportation systems abroad. World Regional Studies, 20(1), 79-87.

Liang, Y., Zheng, X. Q., Bai, S. J., 2016. Study on accessibility of Beijing-Tianjin-Hebei road network using spatial syntax model. Bulletin of Surveying and Mapping, (10), 101-105 
Li, X. J., Wang J. Y., 2017. Study on airport accessibility evaluation based on factor analysis. Technology \& Economy in Areas of Communications, 19(1), 5-8.

Peponis, J., Wineman, J., Rashid, M., Kim, S., Bafna, S., 1997. On the description of shape and spatial configuration inside buildings: convex partitions and their local properties. Environment \& Planning B Planning \& Design, 24(5), 457-462.

Shao, R. Q., 2010. Improvement of spatial unit segmentation method in grid road network city application of spatial syntactic axis map. Urban Planning Internationalg, 25(2), 62-67.

Sheng, L. F., 2012. Research on coupled development of rail transit and urban space based on spatio-temporal perspective. Beijing Jiaotong University, Beijing, China.

Wang, S., Wang, B. W., 2013. Axis map analysis of facility network and place network in pedestrian community --- taking the survey of the elderly community in Anshan Street Community in Shanghai as an example. Huazhong Architecture, (2), 77-82.

Yang, J. W., Zhou, Y. X., 1999. Accessibility: Concept, measurement and application. Geography and Geo-information Science, (2), 61-66.

Zheng, X. Q., Su, Y. J., Yang, G., Wang, S. Q., 2008. Research methods of urban road network complexity: integrated analysis of spatial syntax and complex network model. Summary of Surveying and Mapping Bulletin Frontier Technology Forum.

Zhang, S. G., Wang, C. S., Xu, J. C., 2008 Reachability Study of road network based on time impedance function. Progress in Geography, 27(4), 117-121.

Zhai, Y. F., 2008. Application of modern space syntax theory in residential garden space research. Zhejiang University, Hangzhou, Zhejiang province, China. 